\title{
Magnetic and thermal properties of a one-dimensional spin-1 model
}

\author{
F.Mancini ${ }^{1}$, F.P.Mancini ${ }^{2}$ \\ 1 Dipartimento di Fisica "E. R. Caianiello" - Unità CNISM di Salerno, \\ Università degli Studi di Salerno, Via S. Allende, I-84081 Baronissi (SA), Italy \\ 2 Dipartimento di Fisica and Sezione I.N.F.N., Università degli Studi di Perugia, \\ Via A. Pascoli, I-06123 Perugia, Italy
}

Received May 27, 2008

\begin{abstract}
We study the one-dimensional $S=1$ Blume-Emery-Griffiths model. Upon transforming the spin model into an equivalent fermionic model, we provide the exact solution within the Green's function and equations of motion formalism. We show that the relevant response functions as well as thermodynamic quantities can be determined, in the whole parameters space, in terms of a finite set of local correlators. Furthermore, considering the case of an antiferromagnetic chain with single-ion anisotropy in the presence of an external magnetic field, we find three plateaus in the magnetization curve. In the neighborhood of the endpoints of the intermediate plateau, the specific heat shows a double peak structure.
\end{abstract}

Key words: Blume-Emery-Griffiths model, magnetic plateau, exact solution

PACS: $75.10 . P q, 75.10 .-b, 75.30 . K z$

\section{Introduction}

The spin-1 Ising model with bilinear $(J)$ and biquadratic $(K)$ nearest-neighbor pair interactions and a single-ion potential $(\Delta)$ is known as the Blume-Emery-Griffiths (BEG) model [1]. With vanishing biquadratic interactions, the model is known as the Blume-Capel model [2,3].

There is a large and diffused interest in the study of this model, motivated by several reasons. The BEG model was originally introduced to describe the phase separation and superfluidity in the ${ }^{3} \mathrm{He}-{ }^{4} \mathrm{He}$ mixtures, but it can also describe the properties of a variety of systems ranging from spin-1 magnets to liquid crystal mixtures, microemulsions, semiconductor alloys, to quote a few. Both the BEG and BC models have been investigated using many different approaches for different lattice types and dimensions. In one dimension and zero magnetic field, the spin-1 Ising model and the BEG model have been solved exactly by means of the transfer matrix method [4] and by means of the Bethe method [5]. Exact solutions have also been obtained for a Bethe lattice [6] and for the two-dimensional honeycomb lattice [7]. For higher dimensions, among the various approximate and simulation techniques, the most common approach to the BC and BEG models is based on the use of mean field approximation. However, renormalization group studies show some qualitative differences from the mean field results. The one-dimensional case for the BEG model was studied in [8], where exact renormalization-group recursion relations were derived, exhibiting tricritical and critical fixed points. We refer the interested reader to [9] for a rather broad list of works devoted to the study of the BC and BEG models.

In previous works [10], we have shown that, upon transforming to fermionic variables, spin systems can be conveniently studied by means of quantum field methods, namely: Green's functions and equations of motion methods. This approach has the advantage of offering a general formulation for any dimension and to provide a rigorous determination of a complete set of eigenoperators of the Hamiltonian and, correspondingly, of the set of elementary excitations. In this paper we apply this formulation to the 1D BEG model, and we obtain the exact solution of the model in the whole space of the parameters. Postponing to a forthcoming work a comprehensive analysis of the model, 
in this paper we focus our study on the thermal and magnetic properties of the antiferromagnetic $(J<0)$ spin-1 chain with single-ion anisotropy in the presence of an external magnetic field, in the limit of vanishing biquadratic interaction. This particular case is indeed interesting since one finds magnetization plateaus, experimentally observed [12]. At $T=0$ the magnetization curve forms plateaus with abrupt jumps from one to another at certain values of the magnetic field $h$. When $\Delta=0$, the ground state is purely antiferromagnetic for $-2|J|<h<2|J|$. By varying the magnetic field, the system undergoes a phase transition to a pure ferromagnetic regime at $h= \pm 2|J|$. When the anisotropy $\Delta$ is turned on, one observes an intermediate phase characterized by half of the spins oriented along the external field and the rest perpendicular to it. The intermediate phase between the anti- and ferromagnetic ones, has a width depending on $\Delta$ whose endpoints are denoted by $h_{\mathrm{c}}$ and $h_{\mathrm{s}}$, i.e., critical and saturated field, respectively.

The paper is organized as follows. In section 2, upon introducing a complete set of composite operators, eigenoperators of the Hamiltonian, we outline the analysis leading to the algebra closure and to analytical expressions of the retarded Green's functions (GFs) and correlation functions (CFs). Since the composite operators do not satisfy a canonical algebra, the GF and CF depend on a set of internal parameters, leading only to exact relations among the CFs. According to the scheme of a composite operator method [11], it is possible to determine these parameters by means of algebra constraints fixing the representation of the GF. By following this scheme, in section 3 we obtain extra equations closing the set of relations and allowing for an exact and complete solution of the 1D BEG model. Section 4 is devoted to the study of the finite temperature properties. Finally, section 5 is devoted to our conclusions and final remarks, while the appendix reports some relevant computational details.

\section{Composite fields and Green functions}

The Blume-Emery-Griffiths (BEG) model describes a system with three states per spin. The Hamiltonian of the one-dimensional BEG model with first-nearest neighbor interaction is given by

$$
H=-J \sum_{i} S(i) S(i+1)-K \sum_{i} S^{2}(i) S^{2}(i+1)+\Delta \sum_{i} S^{2}(i)-h \sum_{i} S(i),
$$

where the spin variable $S(i)$ takes the values $S(i)=-1,0,1$. We use the Heisenberg picture: $i=(i, t)$, where $i$ stands for the lattice vector $R_{i}$. This model can be mapped into a fermionic model by means of the transformation

$$
S(i)=[n(i)-1]
$$

where $n(i)=\sum_{\sigma} c_{\sigma}^{\dagger}(i) c_{\sigma}(i)=c^{\dagger}(i) c(i)$ is the density number operator of a fermionic system; $c(i)$ $\left(c^{\dagger}(i)\right)$ is the annihilation (creation) operator of fermionic field in the spinor notation and satisfies canonical anti-commutation relations. Under the transformation (2), the Hamiltonian (1) takes the form

$$
\begin{aligned}
H= & V \sum_{i} n(i) n^{\alpha}(i)+\tilde{U} \sum_{i} D(i)+\frac{1}{2} W \sum_{i} n(i) D^{\alpha}(i) \\
& +\frac{1}{2} W \sum_{i} D(i) n^{\alpha}(i)-W \sum_{i} D(i) D^{\alpha}(i)-\tilde{\mu} \sum_{i} n(i)+E_{0}
\end{aligned}
$$

where we have defined

$$
\begin{aligned}
V & =-(J+K), & & \tilde{U}=2(-2 K+\Delta), \\
W & =4 K, & & \tilde{\mu}=-(2 J+2 K-\Delta \\
E_{0} & =(-J-K+\Delta+h) N . & &
\end{aligned}
$$

Hereafter, for a generic operator $\Phi(i)$ we shall use the following notation: $\Phi^{\alpha}(i)=[\Phi(i+1)+$ $\Phi(i-1)] / 2$. The Hamiltonian (3) is pertinent to an Hubbard model extended to include intersite 
interactions, namely: charge-charge $(V)$, charge-double occupancy $(W)$ and double occupancydouble occupancy $(-W)$ interactions. However, Hamiltonian (3) does not exactly correspond to the BEG Hamiltonian (1) since the mapping between $S$ and $n$ should take into account the four possible values of the particle density $\left(n(i)=0, n_{\uparrow}(i)=1\right.$ and $\left.n_{\downarrow}(i)=1, n(i)=2\right)$. Letting the zero-state spin be degenerate, makes the Hamiltonians (1) and (3) equivalent, provided one redefines the chemical potential $\tilde{\mu}$ and the onsite potential $\tilde{U}$ as

$$
\tilde{\mu} \rightarrow \mu=\tilde{\mu}-\beta^{-1} \ln 2, \quad \tilde{U} \rightarrow U=\tilde{U}-2 \beta^{-1} \ln 2,
$$

where $\beta=1 / k_{\mathrm{B}} T$. As a result, for a translationally invariant chain, one has

$$
H=V \sum_{i} n(i) n^{\alpha}(i)+U \sum_{i} D(i)+W \sum_{i} n(i) D^{\alpha}(i)-W \sum_{i} D(i) D^{\alpha}(i)-\mu \sum_{i} n(i)+E_{0}
$$

It is straightforward to verify that the partition functions relative to the two models (1) and (6) are the same. Therefore, in the following we shall consider the fermionic model, described by the Hamiltonian (6), which is exactly equivalent to the BEG model. To solve this Hamiltonian we shall use the formalism of Green's functions and equations of motion. As a first step, we show that there exists a complete set of eigenoperators and eigenvalues of $H$. To this end, one can introduce the Hubbard projection operators

$$
\xi(i)=[1-n(i)] c(i), \quad \eta(i)=n(i) c(i)
$$

These fields satisfy the equations of motion

$$
\begin{aligned}
\mathrm{i} \frac{\partial}{\partial t} \xi(i) & =[\xi(i), H]=\left[-\mu+2 V n^{\alpha}(i)+W D^{\alpha}(i)\right] \xi(i), \\
\mathrm{i} \frac{\partial}{\partial t} \eta(i) & =[\eta(i), H]=\left[-\mu+U+(2 V+2 W) n^{\alpha}(i)-W D^{\alpha}(i)\right] \eta(i) .
\end{aligned}
$$

By noting that the particle density $n(i)$ and double occupancy $D(i)$ operators satisfy the following algebra

$$
\begin{aligned}
n^{p}(i) & =n(i)+a_{p} D(i) \\
D^{p}(i) & =D(i) \\
n^{p}(i) D(i) & =2 D(i)+a_{p} D(i)
\end{aligned} \quad \text { for } \quad p \geqslant 1,
$$

where $a_{p}=2^{p}-2$, one can derive the following recursion rules

$$
\left[n^{\alpha}(i)\right]^{p}=\sum_{m=1}^{4} A_{m}^{(p)}\left[n^{\alpha}(i)\right]^{m}, \quad\left[D^{\alpha}(i)\right]^{p}=\sum_{m=1}^{2} B_{m}^{(p)}\left[D^{\alpha}(i)\right]^{m} .
$$

The coefficients $A_{m}^{(p)}$ and $B_{m}^{(p)}$ are rational numbers, satisfying the sum rules $\sum_{m=1}^{4} A_{m}^{(p)}=1$ and $\sum_{m=1}^{2} B_{m}^{(p)}=1$, whose explicit expressions are given in the appendix.

On the basis of the equations of motion (8) and by means of the recursion rules (10), one can easily infer that the composite multiplet operators

$$
\psi^{(\xi)}(i)=\left(\begin{array}{c}
\xi(i) \\
\xi(i) n^{\alpha}(i) \\
\xi(i)\left[n^{\alpha}(i)\right]^{2} \\
\xi(i)\left[n^{\alpha}(i)\right]^{3} \\
\xi(i)\left[n^{\alpha}(i)\right]^{4} \\
\xi(i) D^{\alpha}(i) \\
\xi(i)\left[D^{\alpha}(i)\right]^{2}
\end{array}\right), \quad \psi^{(\eta)}(i)=\left(\begin{array}{c}
\eta(i) \\
\eta(i) n^{\alpha}(i) \\
\eta(i)\left[n^{\alpha}(i)\right]^{2} \\
\eta(i)\left[n^{\alpha}(i)\right]^{3} \\
\eta(i)\left[n^{\alpha}(i)\right]^{4} \\
\eta(i) D^{\alpha}(i) \\
\eta(i)\left[D^{\alpha}(i)\right]^{2}
\end{array}\right)
$$


are eigenoperators of $H$ :

$$
\begin{aligned}
\mathrm{i} \frac{\partial}{\partial t} \psi^{(\xi)}(i) & =\left[\psi^{(\xi)}(i), H\right]=\varepsilon^{(\xi)} \psi^{(\xi)}(i), \\
\mathrm{i} \frac{\partial}{\partial t} \psi^{(\eta)}(i) & =\left[\psi^{(\eta)}(i), H\right]=\varepsilon^{(\eta)} \psi^{(\eta)}(i) .
\end{aligned}
$$

The energy matrices $\varepsilon^{(\xi)}$ and $\varepsilon^{(\eta)}$ are matrices of rank $7 \times 7$ and have the expressions

$$
\begin{aligned}
& \varepsilon^{(\xi)}=\left(\begin{array}{ccccccc}
-\mu & 2 V & 0 & 0 & 0 & W & 0 \\
0 & -\mu+\frac{W}{6} & 2 V-\frac{1}{2} W & \frac{1}{3} W & 0 & W & 0 \\
0 & \frac{W}{6} & -\mu-\frac{1}{3} W & 2 V-\frac{W}{6} & \frac{1}{3} W & W & 0 \\
0 & -\frac{1}{3} W & \frac{7}{4} W & -\mu-\frac{35}{12} W & 2 V+\frac{3 W}{2} & W & 0 \\
0 & -3 V-\frac{25}{12} W & \frac{25}{2} V+\frac{205}{24} W & -\frac{35}{2} V-\frac{265}{24} W & -\mu+10 V+\frac{55}{12} W & W & 0 \\
0 & \frac{1}{3} V & -V & \frac{2}{3} V & 0 & -\mu+2 V & W \\
0 & -\frac{1}{3} V & \frac{4}{3} V & -\frac{5}{3} V & \frac{2}{3} V & -\frac{W}{2} & -\mu+2 V+\frac{3 W}{2}
\end{array}\right), \\
& \varepsilon^{(\eta)}=\left(\begin{array}{ccccccc}
-(\mu-U) & \Upsilon & 0 & 0 & 0 & -W & 0 \\
0 & U-\mu-\frac{1}{6} W & 2 V+\frac{3 W}{2} & -\frac{1}{3} W & 0 & -W & 0 \\
0 & -\frac{1}{6} W & U-\mu+\frac{1}{3} W & 2 V+\frac{7}{6} W & -\frac{1}{3} W & -W & 0 \\
0 & \frac{1}{3} W & -\frac{7}{4} W & U-\mu+\frac{35}{12} W & 2 V-\frac{W}{2} & -W & 0 \\
0 & \frac{7}{12} W-3 V & \frac{25}{2} V-\frac{55}{24} W & \frac{55}{24} W-\frac{35}{2} V & U-\mu+10 V+\frac{5}{12} W & -W & 0 \\
0 & \frac{1}{6} \Upsilon & -\frac{1}{2} \Upsilon & \frac{1}{3} \Upsilon & 0 & U-\mu+\Upsilon & -W \\
0 & -\frac{1}{6} \Upsilon & \frac{2}{3} \Upsilon & -\frac{5}{6} \Upsilon & \frac{1}{3} \Upsilon & \frac{W}{2} & U-\mu+2 V-\frac{1}{2} W
\end{array}\right),
\end{aligned}
$$

where $\Upsilon=2 V+W$. The energy levels are given by the eigenvalues of the energy matrices and are as follows:

$$
E_{n}^{(\xi)}=\left(\begin{array}{c}
-\mu \\
-\mu+V \\
-\mu+2 V \\
-\mu+W+2 V \\
-\mu+W / 2+2 V \\
-\mu+W+4 V \\
-\mu+W / 2+3 V
\end{array}\right), \quad E_{n}^{(\eta)}=\left(\begin{array}{c}
-\mu+U \\
-\mu+U+W / 2+V \\
-\mu+U+W+2 V \\
-\mu+U+2 V \\
-\mu+U+W / 2+2 V \\
-\mu+U+W+4 V \\
-\mu+U+W+3 V
\end{array}\right)
$$

The knowledge of a complete set of eigenoperators and eigenvalues of the Hamiltonian allows for an exact expression of the retarded Green's function

$$
G^{(s)}\left(t-t^{\prime}\right)=\theta\left(t-t^{\prime}\right)\left\langle\left\{\psi^{(s)}(i, t), \psi^{(s)^{\dagger}}\left(i, t^{\prime}\right)\right\}\right\rangle=\frac{\mathrm{i}}{(2 \pi)} \int_{-\infty}^{+\infty} \mathrm{d} \omega \mathrm{e}^{-\mathrm{i} \omega\left(t-t^{\prime}\right)} G^{(s)}(\omega)
$$

and, consequently, of the correlation function

$$
C^{(s)}\left(t-t^{\prime}\right)=\left\langle\psi^{(s)}(i, t) \psi^{(s)^{\dagger}}\left(i, t^{\prime}\right)\right\rangle=\frac{1}{(2 \pi)} \int_{-\infty}^{+\infty} \mathrm{d} \omega \mathrm{e}^{-\mathrm{i} \omega\left(t-t^{\prime}\right)} C^{(s)}(\omega) .
$$

In the above equations $s=\xi, \eta$ and $\langle\cdots\rangle$ denotes the quantum-statistical average over the grand canonical ensemble. One finds

$$
\begin{aligned}
G^{(s)}(\omega) & =\sum_{n=1}^{7} \frac{\sigma^{(s, n)}}{\omega-E_{n}^{(s)}+\mathrm{i} \delta}, \\
C^{(s)}(\omega) & =\pi \sum_{n=1}^{7} \sigma^{(s, n)} T_{n}^{(s)} \delta\left(\omega-E_{n}^{(s)}\right),
\end{aligned}
$$


where $T_{n}^{(s)}=1+\tanh \left(\beta E_{n}^{(s)} / 2\right)$ and the spectral density matrices $\sigma^{(s, n)}$ are computed by means of the formula

$$
\sigma_{\mu \nu}^{(s, n)}=\Omega_{\mu n}^{(s)} \sum_{\lambda=1}^{7}\left[\Omega_{n \lambda}^{(s)}\right]^{-1} I_{\lambda \nu}^{(s)},
$$

where $\Omega^{(s)}$ is the $7 \times 7$ matrix whose columns are the eigenvectors of the matrix $\varepsilon^{(s)}$. The explicit expressions of the spectral density matrices are given in the appendix. $I^{(s)}$ is the normalization matrix defined as

$$
I^{(s)}=\left\langle\left\{\psi^{(s)}(i), \psi^{(s)^{\dagger}}(i)\right\}\right\rangle .
$$

By means of the recurrence relations (10), all the matrix elements of $I^{(s)}$ can be expressed in terms of only the elements belonging to the first row. The calculations of the latter give, for a homogeneous state,

$$
\begin{aligned}
& I_{1, k}^{(\xi)}=\kappa^{(k-1)}-\lambda^{(k-1)}, \quad(k=1, \ldots 5), \quad I_{1, k}^{(\xi)}=\delta^{(k-5)}-\theta^{(k-5)}, \quad(k=6,7), \\
& I_{1, k}^{(\eta)}=\lambda^{(k-1)}, \quad(k=1, \ldots 5), \quad I_{1, k}^{(\eta)}=\theta^{(k-5)}, \quad(k=6,7),
\end{aligned}
$$

where

$$
\begin{aligned}
\kappa^{(p)} & =\left\langle\left[n^{\alpha}(i)\right]^{p}\right\rangle, & \delta^{(p)} & =\left\langle\left[D^{\alpha}(i)\right]^{p}\right\rangle, \\
\lambda^{(p)} & =\frac{1}{2}\left\langle n(i)\left[n^{\alpha}(i)\right]^{p}\right\rangle, & \theta^{(p)} & =\frac{1}{2}\left\langle n(i)\left[D^{\alpha}(i)\right]^{p}\right\rangle .
\end{aligned}
$$

In conclusion, in this section we have shown that the $1 \mathrm{D}$ BEG model is exactly solvable. Exact expressions for the GF and CF have been obtained and are expressed in terms of a set of local correlation functions (24), which should be calculated in order to obtain quantitative results. This problem will be considered in the next section, where a self-consistent scheme, capable of computing the internal parameters, will be formulated.

\section{Self-consistent equations}

Based on the computational framework provided in the previous section, it is evident that the GF and the CF depend on the internal parameters: $\mu, \kappa^{(p)}$ and $\lambda^{(p)}(p=0, \ldots, 4), \delta^{(p)}$ and $\theta^{(p)}(p=1,2)$. For a homogeneous state (i.e., translationally invariant: $\left\langle n^{\alpha}(i)\right\rangle=\langle n(i)\rangle$ and $\left.\langle D(i)\rangle=\left\langle D^{\alpha}(i)\right\rangle\right)$, there are twelve parameters to be self-consistently computed in terms of the external parameters $n, V, U, W$ and $T$. A first set of self-consistent equations is given by the algebra constraints

$$
\begin{array}{lll}
\xi_{\uparrow}(i) \xi_{\uparrow}^{\dagger}(i)+\eta_{\uparrow}(i) \eta_{\uparrow}^{\dagger}(i)=1-n_{\uparrow}(i), & & \eta_{\uparrow}(i) \eta_{\uparrow}^{\dagger}(i)=n_{\downarrow}(i)-D(i), \\
\xi_{\downarrow}(i) \xi_{\downarrow}^{\dagger}(i)+\eta_{\downarrow}(i) \eta_{\downarrow}^{\dagger}(i)=1-n_{\downarrow}(i), & & \eta_{\downarrow}(i) \eta_{\downarrow}^{\dagger}(i)=n_{\uparrow}(i)-D(i)
\end{array}
$$

from which one gets the following self-consistent equations

$$
\begin{array}{ll}
C_{1,1}^{(\eta)}=\lambda^{(0)}-\delta^{(1)} & \\
C_{1, k}^{(\xi)}+C_{1, k}^{(\eta)}=\kappa^{(k-1)}-\lambda^{(k-1)}, & (k=1, . .5), \\
C_{1, k}^{(\xi)}+C_{1, k}^{(\eta)}=\delta^{(k-5)}-\theta^{(k-5)}, & (k=6,7),
\end{array}
$$

where the CFs in the l.h.s. of equation (26) can be computed by means of the formula

$$
C^{(s)}=\left\langle\psi^{(s)}(i) \psi^{(s)^{\dagger}}(i)\right\rangle=\frac{1}{2} \sum_{n=1}^{7} \sigma^{(s, n)} T_{n}^{(s)} .
$$


Equations (26) provide one with eight self-consistent equations. To determine all the parameters one needs other four equations. These can be derived by means of the algebra constraints

$$
\xi^{\dagger}(i) n(i)=0, \quad \xi^{\dagger}(i) D(i)=0
$$

By exploiting these relations one can express the CFs $C_{13}^{(\xi \xi)}, C_{14}^{(\xi \xi)}, C_{15}^{(\xi \xi)}$, and $C_{17}^{(\xi \xi)}$ in terms of the $\mathrm{CFs} C_{11}^{(\xi \xi)}, C_{12}^{(\xi \xi)}$ and $C_{16}^{(\xi \xi)}$ as

$$
\begin{aligned}
C_{13}^{(\xi \xi)} & =C_{11}^{(\xi \xi)}\left(\frac{1}{2} X_{1}+X_{2}+\frac{1}{2} X_{1}^{2}\right) \\
C_{14}^{(\xi \xi)} & =C_{11}^{(\xi \xi)}\left(\frac{1}{4} X_{1}+\frac{3}{2} X_{2}+\frac{3}{2} X_{1} X_{2}+\frac{3}{4} X_{1}^{2}\right) \\
C_{15}^{(\xi \xi)} & =C_{11}^{(\xi \xi)}\left(\frac{1}{8} X_{1}+\frac{7}{4} X_{2}+\frac{9}{2} X_{1} X_{2}+\frac{7}{8} X_{1}^{2}+\frac{3}{2} X_{2}^{2}\right) \\
C_{17}^{(\xi \xi)} & =C_{11}^{(\xi \xi)}\left(\frac{1}{2} X_{2}+\frac{1}{2} X_{2}^{2}\right)
\end{aligned}
$$

The two parameters $X_{1}$ and $X_{2}$ are expressed in terms of the CFs $C_{11}^{(\xi \xi)}, C_{12}^{(\xi \xi)}$ and $C_{16}^{(\xi \xi)}$ as

$$
X_{1}=\frac{C_{12}^{(\xi \xi)}}{C_{11}^{(\xi \xi)}}, \quad X_{2}=\frac{C_{16}^{(\xi \xi)}}{C_{11}^{(\xi \xi)}} .
$$

Equations (26), (29) and (30) provide twelve self-consistent equations which will determine all the unknown internal parameters and therefore the various properties of the model. Once the parameters of the fermionic model are computed, by use of the mapping transformations (4) and (5), it is straightforward to study the behavior of relevant properties of the BEG model. Details of the computations leading to equation (29) will be given elsewhere [16].

\section{Magnetic and thermal responses}

As an application of the general formulation provided in the previous sections, here we shall study the magnetic and thermal properties of the model Hamiltonian (1), by restricting the analysis to the case $K=0$ and $J<0$. In the following we set $J=-1$ and we consider only positive values of $h$, owing to the symmetry property of the model under the transformation $h \rightarrow-h$. When $\Delta=0$, the ground state is either antiferromagnetic or ferromagnetic, depending on the value of the external field. As a consequence, the magnetization, defined as

$$
m=\langle S(i)\rangle=\langle n(i)\rangle-1=1-2\left[C_{11}^{(\xi)}+C_{11}^{(\eta)}\right]
$$

presents, at $T=0$, two plateaus as a function of the external field $h$, with $h_{\mathrm{s}}=2$ being the value of the saturated field. Turning on a positive single-ion anisotropy, three plateaus appear at $m=0$, $m=1 / 2$ and $m=1$. This is in agreement with the criterion derived in [13] for the appearance of plateaus in spin chains in a uniform magnetic field.

In figure $1 \mathrm{a}$ we plot the magnetization as a function of the magnetic field at $T=0.01$ for different values of $\Delta$. Upon increasing the field, a nonzero magnetization begins at the critical value of the field $h_{\mathrm{c}}$ : this critical value decreases by increasing $\Delta$. For $h>h_{\mathrm{c}}$, a $m=1 / 2$ plateau is observed until $h$ reaches the saturated value $h_{\mathrm{s}}$, at which the third magnetization plateau at $m=1$ is observed. The width of the $m=1 / 2$ plateau augments by increasing $\Delta$ in the range $0<\Delta<1$ and becomes independent of $\Delta$ when $\Delta>1$. The critical field $h_{\mathrm{c}}$ and the saturated field $h_{\mathrm{s}}$ satisfy, in the range $0<\Delta<1$, the laws:

$$
h_{\mathrm{c}}=2-\Delta, \quad h_{\mathrm{s}}=2+\Delta .
$$




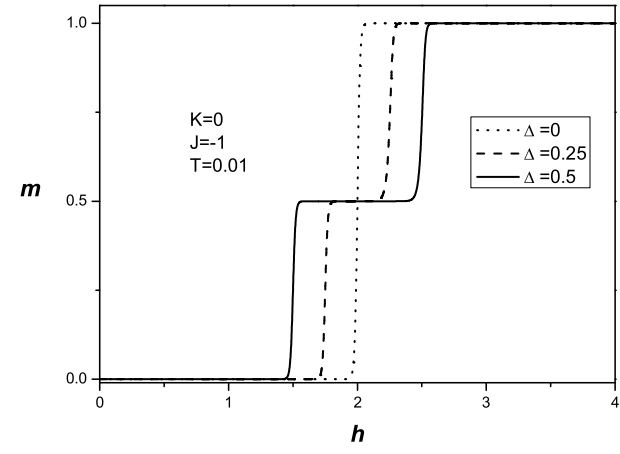

(a)

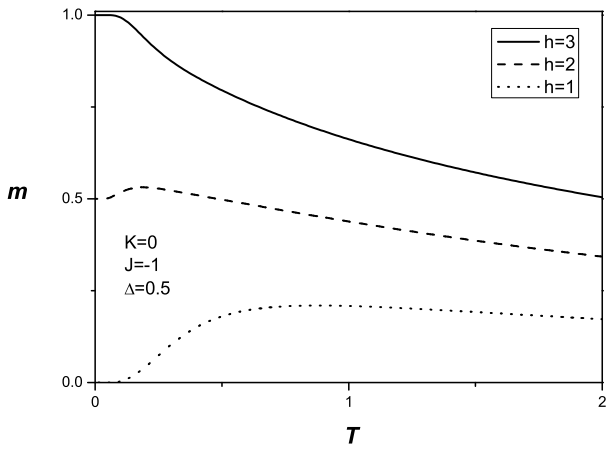

(b)

Figure 1. (a) The magnetization $m$ as a function of the external field $h$ for $K=0, J=-1$, $T=0.01$ and for $\Delta=0,0.5$ and 1. (b) The magnetization as a function of the temperature for $K=0, J=-1, \Delta=0.5$ and $h=1,2$ and 3 .

Our findings are in good agreement with Monte Carlo [14] and transfer matrix [15] results. In figure $1 \mathrm{~b}$ we plot the magnetization as a function of the temperature for values of the magnetic field belonging to the three different plateaus. For $h=1\left(h<h_{\mathrm{c}}\right)$ the magnetization is zero at $T=0$; all spins are aligned (upward and downward) with the magnetic field, resulting in a pure AF state. When the temperature increases, the thermal fluctuations allow some of the downward spins to rotate and the magnetization increases up to $T \approx 0.9$ where it exhibits a maximum. Further increasing $T$, the thermal fluctuations enter in competition with the magnetic field and the magnetization decreases. For $h=2$ (intermediate phase $h_{\mathrm{c}}<h<h_{\mathrm{s}}$ ) the magnetization is equal to $1 / 2$ at $T=0$ : half of the spins are parallel to $h$ and half of them lie in the transverse plane. When the temperature increases, there is a slight increase of $m$ (up to $T \approx 0.2$ ), but soon the disorder induced by thermal fluctuation prevails and $m$ decreases. For $h=3\left(h>h_{\mathrm{s}}\right)$ at $T=0$ one finds $m=1$ : all spins are parallel to the magnetic field and the system is in a pure ferromagnetic state. When the temperature increases, the long-range order is destroyed and the magnetization decreases.

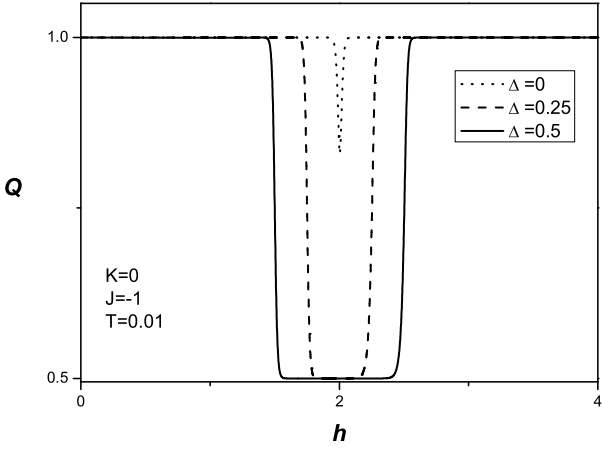

(a)

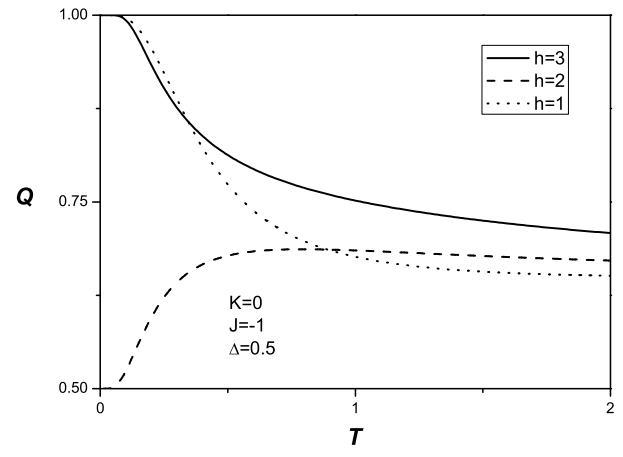

(b)

Figure 2. (a) The quadrupolar moment $Q$ as a function of the external magnetic field $h$ for $K=0, J=-1, T=0.01$ and for $\Delta=0,0.5$ and 1. (b) The quadrupolar moment as a function of the temperature for $K=0, J=-1, \Delta=0.5$ and $h=1,2$ and 3 . 
To further analyze the magnetic behavior, we have studied the quadrupolar moment $Q$, defined as

$$
Q=\left\langle S^{2}(i)\right\rangle=1-2 C_{11}^{(\eta)}
$$

At zero temperature, this quantity also shows plateaus for $\Delta \geqslant 0$. In figure 2 a the quadrupolar moment $Q$ is plotted as a function of the external magnetic field, for $K=0, J=-1, T=0.01$ and for various values of $\Delta$. $Q$ takes the value $1 / 2$ in the range $h_{\mathrm{c}}<h<h_{\mathrm{s}}$, whereas it is equal to 1 for all other values of $h$. The behavior of $Q$ as a function of the temperature is shown in figure $2 \mathrm{~b}$. For $h<h_{\mathrm{c}}$ and $h>h_{\mathrm{s}}$, the quadrupolar moment $Q$ is maximum $(Q=1)$ at $T=0$ and decreases by increasing $T$. For $h_{\mathrm{c}}<h<h_{\mathrm{s}}, Q$ vanishes at zero temperature and increases augmenting $T$.

The existence of the magnetic plateaus is endorsed by the peaks found in the magnetic susceptibility $\chi=\mathrm{d} m / \mathrm{d} h$. As evidenced in figure $3 \mathrm{a}$, for $\Delta=0$ the susceptibility presents only one peak when plotted as a function of $h$, whereas for $\Delta>0$ there are two peaks appearing at $h_{\mathrm{c}}$ and $h_{\mathrm{s}}$, signalling a step-like behavior of the magnetization. When plotted as a function of the temperature, $\chi$ shows a peak at low temperatures and then vanishes for $T \rightarrow 0$ for all values of the magnetic field except at $h_{c, s}$, where, of course, it diverges due to the step encountered by the magnetization. As an example, in figure $3 \mathrm{~b}$ we plot the susceptibility in the neighborhood of $h_{\mathrm{s}}$.

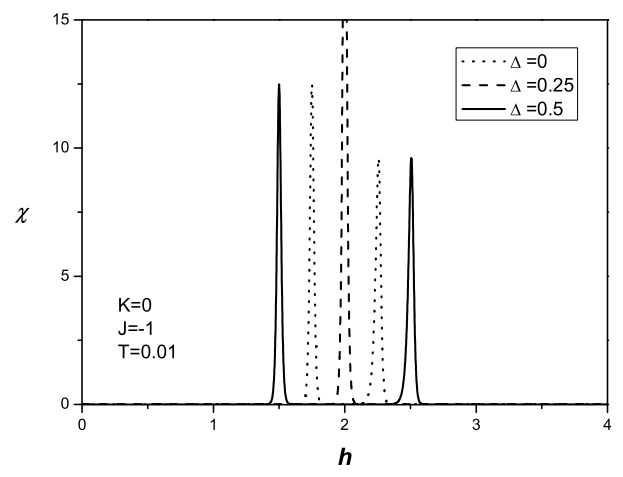

(a)

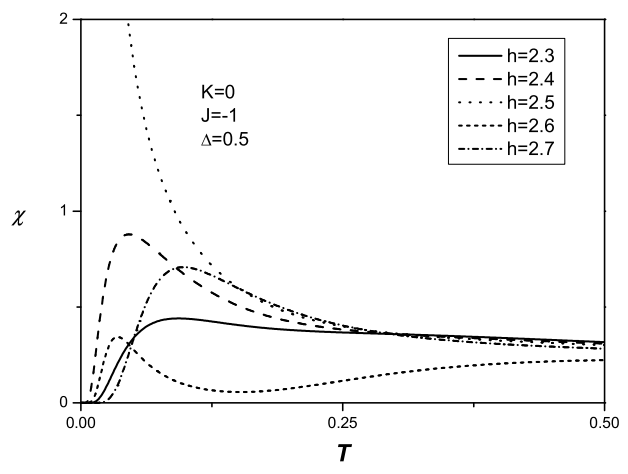

(b)

Figure 3. (a) The susceptibility $\chi$ as a function of the external field for $K=0, J=-1, T=0.01$ and for $\Delta=0,0.5$ and 1 . (b) The susceptibility as a function of the temperature for $K=0$, $J=-1, \Delta=0.5$ in the neighborhood of $h_{\mathrm{s}}$.

The specific heat is given by $C=\mathrm{d} E / \mathrm{d} T$, where the internal energy $E$ can be computed as the thermal average of the Hamiltonian (1) for $K=0$

$$
E=-J\left\langle S(i) S(i)^{\alpha}\right\rangle+\Delta Q-h m
$$

The specific heat exhibits a rich structure in correspondence of the critical values of the magnetic field. The behavior of the specific heat as a function of the temperature is shown in figures $4 a-b$ in the neighborhood of $h_{c, s}$. The possible excitations of the ground state are flipping of the spins parallel to $h$ and/or of the ones perpendicular to it. Away from $h_{\mathrm{c}, \mathrm{s}}$, the specific heat presents only one peak at low temperatures. When $h<h_{\mathrm{c}}$, the possible excitations are due only to the flipping of the longitudinal spins. In the neighborhood of $h_{\mathrm{c}}$, a second peak appears since thermal fluctuations also tend to flip the spins perpendicular to the external field. Further increasing $h$, the specific heat presents only one peak until $h \approx h_{\mathrm{s}}$, where again two peaks are present. Away from $h_{\mathrm{s}}$, the specific heat shows only one peak. 


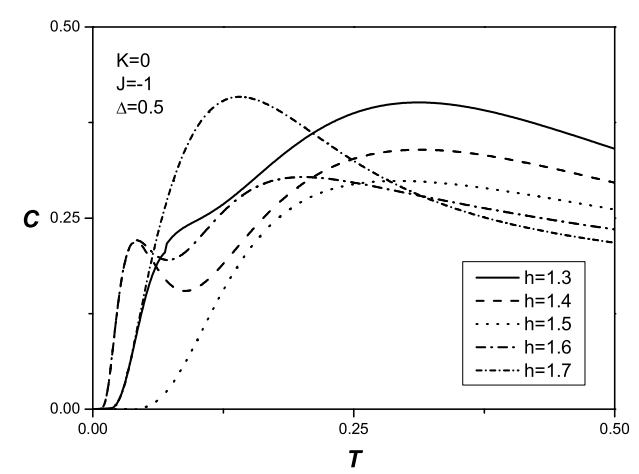

(a)

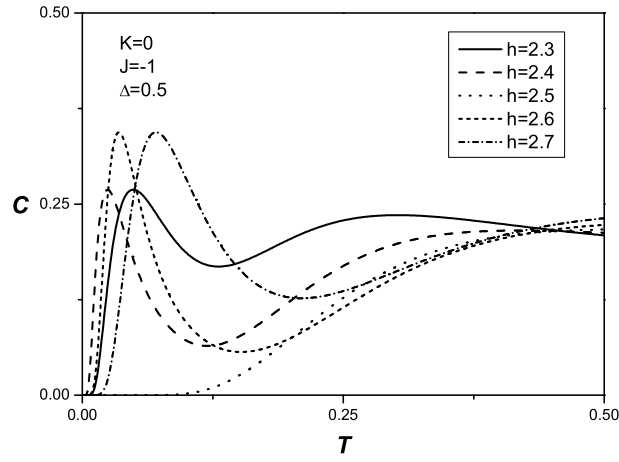

(b)

Figure 4. The specific heat as a function of the temperature for $K=0, J=-1, \Delta=0.5$, (a) around $h_{\mathrm{c}}=1.5 ;(\mathrm{b})$ around $h_{\mathrm{s}}=2.5$.

\section{Concluding remarks}

We have evidenced how the use of the Green's function and equations of motion formalism leads to the exact solution of the one-dimensional BEG model. Our analysis allows for a comprehensive study of the model in the whole space of parameters $K, J, \Delta, h$ and $T$. Here, we have focused on the antiferromagnetic properties exhibited by the model and we have shown that, at zero temperature, the model exhibits three magnetic plateaus when $\Delta>0$. Furthermore, the specific heat shows a double peak structure in the neighborhood of the endpoints of the intermediate plateau.

\section{Acknowledgements}

This paper is dedicated to Professor Ihor Stasyuk on the occasion of his 70th birthday, wishing him many more years of successful and fruitful work.

\section{A. Some analytical expressions}

The coefficients $A_{m}^{(p)}$ and $B_{m}^{(p)}$ in equation (10) are given by:

$$
\begin{aligned}
A_{1}^{(p)} & =-6+2^{3-p}-2^{p-1}+2^{3-p} \cdot 3^{p-1}, \\
A_{2}^{(p)} & =\frac{1}{3 \cdot 2^{p+1}}\left(-104+57 \cdot 2^{p+1}-56 \cdot 3^{p}+11 \cdot 4^{p}\right), \\
A_{3}^{(p)} & =\frac{1}{3 \cdot 2^{p-1}}\left(18-3 \cdot 2^{p+3}+14 \cdot 3^{p}-3 \cdot 4^{p}\right), \\
A_{4}^{(p)} & =\frac{1}{3 \cdot 2^{p-1}}\left(-4+3 \cdot 2^{p+1}-4 \cdot 3^{p}+4^{p}\right), \\
& B_{1}^{(p)}=2^{2-p}-1, \quad B_{2}^{(p)}=2-2^{2-p} .
\end{aligned}
$$

$\sigma^{(s, m)}=\Sigma_{m}^{(s)} \Gamma^{(m)}$ are the spectral density matrices defined in equation $(21) ; \Gamma^{(m)}$ are matrices 
of $\operatorname{rank} 7 \times 7$ :

$$
\begin{aligned}
& \Gamma_{1, k}^{(1)}=\left(\begin{array}{lllllll}
1 & 0 & 0 & 0 & 0 & 0 & 0
\end{array}\right) \\
& \Gamma_{l, k}^{(2)}=\left(\begin{array}{lllllll}
1 & 1 / 2 & 1 / 4 & 1 / 8 & 1 / 16 & 0 & 0
\end{array}\right) \\
& \Gamma_{l, k}^{(3)}=\left(\begin{array}{lllllll}
1 & 1 & 1 & 1 & 1 & 0 & 0
\end{array}\right) \\
& \Gamma_{l, k}^{(4)}=\left(\begin{array}{lllllll}
1 & 1 & 1 & 1 & 1 & 1 & 1
\end{array}\right) \\
& \Gamma_{l, k}^{(5)}=\left(\begin{array}{llllllll}
1 & 1 & 1 & 1 & 1 & 1 / 2 & 1 / 4
\end{array}\right) \\
& \Gamma_{l, k}^{(6)}=\left(\begin{array}{lllllll}
1 & 2 & 4 & 8 & 16 & 1 & 1
\end{array}\right)
\end{aligned}
$$

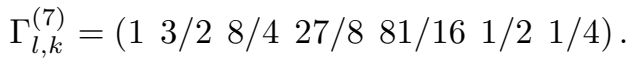

and the $\Sigma_{m}^{(s)}$ are given by:

$$
\begin{aligned}
\Sigma_{1}^{(s)} & =\frac{1}{6}\left(6 I_{1,1}^{(s)}-25 I_{1,2}^{(s)}+35 I_{1,3}^{(s)}-20 I_{1,4}^{(s)}+4 I_{1,5}^{(s)}\right) \\
\Sigma_{2}^{(s)} & =\frac{4}{3}\left(6 I_{1,2}^{(s)}-13 I_{1,3}^{(s)}+9 I_{1,4}^{(s)}-2 I_{1,5}^{(s)}\right) \\
\Sigma_{3}^{(s)} & =-\frac{23}{6} I_{1,2}^{(s)}+\frac{23}{2} I_{1,3}^{(s)}-\frac{26}{3} I_{1,4}^{(s)}+2 I_{1,5}^{(s)}-3 I_{1,6}^{(s)}+2 I_{1,7}^{(s)} \\
\Sigma_{4}^{(s)} & =\frac{1}{6}\left(3 I_{1,2}^{(s)}-11 I_{1,3}^{(s)}+12 I_{1,4}^{(s)}-4 I_{1,5}^{(s)}-6 I_{1,6}^{(s)}+12 I_{1,7}^{(s)}\right) \\
\Sigma_{5}^{(s)} & =\frac{4}{3}\left(-2 I_{1,2}^{(s)}+7 I_{1,3}^{(s)}-7 I_{1,4}^{(s)}+2 I_{1,5}^{(s)}+3 I_{1,6}^{(s)}-3 I_{1,7}^{(s)}\right) \\
\Sigma_{6}^{(s)} & =\frac{1}{6}\left(-3 I_{1,2}^{(s)}+11 I_{1,3}^{(s)}-12 I_{1,4}^{(s)}+4 I_{1,5}^{(s)}\right) \\
\Sigma_{7}^{(s)} & =\frac{4}{3}\left(2 I_{1,2}^{(s)}-7 I_{1,3}^{(s)}+7 I_{1,4}^{(s)}-2 I_{1,5}^{(s)}\right)
\end{aligned}
$$

Here we have reported only the first row of the spectral density matrices. All the other matrix elements can be expressed in terms of the first row by means of the recursion relation (10).

\section{References}

1. Blume M., Emery V.J., Griffiths R.B., Phys. Rev. A, 1971, 4, 1071.

2. Blume M., Phys. Rev., 1966, 141, 517.

3. Capel H.W., Physica, 1966, 32, 966; ibid., 1967, 33, 295; ibid., 1967, 37, 423.

4. Suzuki M., Tsujiyama B., Katsura S., J. Math. Phys., 1967, 8, 124; Hintermann A., Rys F., Helv. Phys. Acta, 1969, 42, 608.

5. Obokata T., Oguchi T., J. Phys. Soc. Jpn, 1968, 25, 322.

6. Chakraborty K.G., Tucker J.W., J. Magn. Magn. Mat., 1986, 54-57, 1349.

7. Rosengren A., Haggkvist R., Phys. Rev. Lett., 1989, 63, 660.

8. Krinsky S., Furman D., Phys. Rev. B, 1975, 11, 2602.

9. Mancini F., Eur. Phys. J. B, 2005, 47, 527.

10. Mancini F., Europhys. Lett., 2005, 70, 484; Mancini F., Condens. Matter Phys., 2006, 9, 393; Mancini F., Mancini F.P., Phys. Rev. E, 2008, 77, 061120.

11. Mancini F., Avella A., Adv. Phys., 2004, 53, 537.

12. Narumi Y., Hagiwara M., Sato R., Kindo K., Nakano H., Takahashi M., Physica B, 1998, 246, 509.

13. Oshikawa M., Yamanaka M., Affleck I., Phys. Rev. Lett., 1997, 78, 1984.

14. Chen X.Y., Jiang Q., Shen W.Z., Zhong C.G., J. Magn. Magn. Mat., 2003, 262, 258.

15. Aydiner E., Akyüz C., Chin. Phys. Lett., 2005, 22, 2382.

16. Mancini F., Mancini F.P., in preparation. 


\section{Магнітні та теплові властивості одновимірної спін-1 моделі}

Ф.Манчіні ${ }^{1}$, Ф.П.Манчіні ${ }^{2}$

1 Факультет фізики ім. Е.Р. Каяніелло, Університет Салерно, Бароніссі 84081, Італія

2 Факультет фізики та відділення Національного інституту ядерних досліджень, Університет Перуджі, Перуджа 06123, Італія

Отримано 27 травня 2008 р.

Досліджується одновимірна $S=1$ модель Блюма-Емері-Гріфітса. Після перетворення спінової моделі у еквівалентну ферміонну модель, отримано точний розв'язок в рамках формалізму функцій Ґріна та рівнянь руху. Показано, що для всіх значень параметрів відповідні функції відгуку і термодинамічні величини можуть бути визначені через скінчений набір локальних кореляторів. Крім того, при розгляді одновимірного антиферомагнітного ланцюжка з одноіонною анізотропією, який поміщений у зовнішнє магнітне поле, отримано три плато на кривій намагнічення, а поблизу країв середнього плато питома теплоємність проявляє двопікову структуру.

Ключові слова: модель Блюма-Емері-Гріфітса, magnetic plateau, exact solution

PACS: $75.10 . P q, 75.10 .-b, 75.30 . K z$ 
